\title{
Síndrome da cauda equina em paciente jovem: um estudo de caso
}

\author{
Equine tail syndrome in a young patient: a case study
}

Síndrome de la cola equina en un paciente joven: un estudio de caso

Mariana de Oliveira Lima ${ }^{1 *}$, Beatriz Guedes Borges de Proença1, Daniela Baldo de Oliveira Lima ${ }^{1}$, Marina Rodrigues de Oliveira ${ }^{1}$, Sinésio Grace Duarte ${ }^{1}$.

\begin{abstract}
RESUMO
Objetivo: Relatar um caso de hérnia de disco em paciente feminino jovem, que evoluiu com Síndrome da Cauda Equina (SCE) e necessidade de abordagem cirúrgica imediata. Detalhamento do caso: Paciente feminina, 15 anos, encaminhada ao hospital de referência da cidade de Franca-SP, com quadro de dor lombar modera há 3 semanas e piora nos últimos 15 dias, irradiada para membros inferiores - predominantemente à direita e evoluiu com disfunção urinaria e fecal. A Ressonância Magnética (RM) da coluna lombossacra evidenciou hérnia discal L5S1. Paciente foi submetida imediatamente a laminectomia bilateral e artrodese com estabilização do quadro. A coleta de informações foi autorizada pela paciente e seu responsável legal através do termo de consentimento livre e esclarecido (TCLE) o qual foi subjugado e aceito pelo comitê de ética da fundação Santa Casa de Misericórdia de Franca com o CAAE 00474918.60000.5438. Considerações finais: A SCE é uma emergência neurocirúrgica cujo tratamento cirúrgico tem como objetivo o alívio da dor, a recuperação e preservação neurológica e o restabelecimento funcional.
\end{abstract}

Palavras-chave: Síndrome da cauda equina, Hérnia Discal, Incontinência fecal, Incontinência urinária.

\begin{abstract}
Objective: To report a case of disc herniation in a young female patient who evolved with Cauda Equina Syndrome (CES) and the need for an immediate surgical approach. Detailing of the case: Female patient, 15 years old, referred to the reference hospital of the city of Franca-SP, with low back pain moderates for 3 weeks and worsens in the last 15 days, irradiated to the lower limbs - predominantly to the right and evolved with urination and fecal dysfunction. Magnetic resonance imaging (MRI) of the lumbosacral spine showed L5-S1 disc hernia. The patient was immediately submitted to bilateral laminectomy and arthrodesis with stabilization of the condition. The collection of information was authorized by the patient and her legal guardian through the informed consent form (TCLE), which was subdued and accepted by the ethics committee of the Santa Casa de Misericórdia de Franca foundation with CAAE 00474918.60000.5438. Final considerations: The SCE is a neurocirugic emergency whose surgical treatment aims at pain relief, recovery and neurological preservation and functional restoration.
\end{abstract}

Key words: Cauda equina syndrome, Disc herniation, Fecal incontinence, Urinary incontinence.

\section{RESUMEN}

Objetivo: Reportar un caso de hernia discal en una paciente joven, que evolucionó con Síndrome de Cola Equina (SCE) y la necesidad de un abordaje quirúrgico inmediato. Detalle del caso: Paciente, mujer de 15 años, remitida al hospital de referencia de la ciudad de Franca-SP, con lumbalgia moderada durante 3 semanas y empeorando en los últimos 15 días, irradiando a miembros inferiores - predominantemente en el derecho y evolucionó con disfunción urinaria y fecal. La resonancia magnética (IRM) de la columna lumbosacra mostró una hernia de disco L5-S1. El paciente fue inmediatamente sometido a laminectomy bilateral y artrodesis con estabilización del cuadro. La recopilación de información fue autorizada por la paciente y su tutor legal a través del formulario de consentimiento informado (TCLE), que fue sometido y aceptado por el comité de ética de la fundación Santa Casa de Misericórdia de Franca con CAAE 00474918.60000.5438. Consideraciones finales: EI SCE es una urgencia neuroquirúrgica cuyo tratamiento quirúrgico tiene como objetivo el alivio del dolor, la recuperación y preservación neurológica y la restauración funcional.

Palabras clave: Síndrome de cola de caballo, Hernia de disco, Incontinencia fecal, Incontinencia urinaria.

1 Universidade de Franca (UNIFRAN), Franca - SP. *E-mail: marianaoliveira_70@hotmail.com 


\section{INTRODUÇÃO}

A Síndrome da Cauda Equina (SCE) descreve a condição clínica resultante da neuropatia de múltiplas raízes nervosas lombossacrais (PEREIRA CU, et al., 2013). A SCE é uma patologia com baixa incidência na população geral, com 1 caso para cada 100.000 habitantes, porém observa-se sua ocorrência em qualquer faixa etária, contudo, é mais comum em adultos entre a quarta e quinta década de vida e são escassos os casos descritos na literatura na população jovem (VIALLE LR, et al., 2010). Apesar de rara, suas sequelas são causas de diminuição da qualidade de vida dos pacientes e de altos gastos a saúde pública (VIALLE LR, et al., 2010).

A patogênese dessa doença ainda permanece em investigação, porém observa-se duas hipóteses principais, as quais giram em torno da compressão mecânica ou da isquemia à cauda equina e, observa-se que a compressão anatômica de raízes nervosas, geralmente por uma hérnia de disco vertebral em região lombar é uma causa comum na população geral (YANG SD, et al., 2017).

Dentre outras etiologias, destacam-se: neoplasia espinhal (intradural ou extradural), hematoma, espondilite anquilosante, fratura lombar, osteomielite ou abcesso espinhal, cisto sinovial lombar, terapia manipulativa da coluna vertebral e complicação cirúrgica da coluna lombar (FUSO FA, et al., 2013). Entre os fatores desencadeantes da síndrome estão causas traumáticas e não traumáticas, entretanto a hérnia discal lombar extrusa tem sido considerada uma das principais etiologias (GARDNER A, et al., 2011). As SCE causadas por tumores como linfomas ou por traumas apresentam um diagnóstico difícil (GARDNER A, et al., 2011).

É uma doença que se inicia com episódios de dor lombar isolada, seguida pelo aparecimento de dor ciática que irradiada para um ou ambos os membros inferiores e anestesia em sela, não sendo necessária a presença das três características para confirmação diagnóstica (CHAU AM, el al., 2014). Relacionam-se tais sintomas com fatores precipitantes como um evento traumático, ou como carregar peso ou com algum movimento brusco (CHAU AM, et al., 2014). A dor geralmente piora após atividade física ou algum tempo sentado e é aliviada com o decúbito lateral, na posição de semi-Fowler, em que se inclina o leito a $45^{\circ}$ e flexiona as pernas do paciente (AHN UM, et al., 2011).

Dentre as características clínicas da SCE incluem-se anestesia na região da sela, déficit motor e/ou sensorial, incontinência urinária ou fecal e disfunção sexual (PEREIRA CU, et al., 2013). A condição pode progredir para incontinência permanente, paraplegia ou ambos e a exacerbação do quadro pode ocorrer após o indivíduo alongar-se, tossir ou espirrar (AHN UM, et al., 2011). Pode-se observar fraqueza e parestesia em um ou ambos os membros inferiores, que em geral são transitórias e respeitam um trajeto determinado pelo dermátomo e pelo miótomo correspondentes à raiz comprimida pela herniação (PEREIRA CU, et al., 2013).

O diagnóstico da SCE é baseado na história clínica e no exame neurológico do paciente e os exames de imagem, especialmente a Ressonância Magnética, são usados para confirmar o diagnóstico, a natureza e a localização da lesão (GITELMAN A, et al., 2008). O tratamento instituído é a cirurgia e sua realização precoce, dentro de 48 horas, está associada a melhores desfechos clínicos (CROCKER M, et al., 2008).

Desde modo, é necessário relatar os casos encontrados na prática clínica, a fim de compartilhar experiências e enriquecer as discussões de evolução terapêutica nessa população. Visto isso, o objetivo desse trabalho é relatar um caso de hérnia de disco em uma paciente do sexo feminino, jovem, que evoluiu com SCE com necessidade de abordagem cirúrgica imediata, visando estabilizar a progressão da síndrome e diminuição de sequelas permanentes para a paciente.

\section{DETALHAMENTO DE CASO}

Os dados presentes neste relato foram obtidos através da revisão de prontuário e consulta aos laudos médicos dos meios diagnósticos pelos quais a paciente foi submetida, sendo a coleta de informações autorizada pela paciente e seu responsável legal através do termo de consentimento livre e esclarecido (TCLE) o qual foi subjugado e aceito pelo comitê de ética da fundação Santa Casa de Misericórdia de Franca com o 
CAAE 00474918.60000.5438, e revisão de literatura, por meio de artigos científicos nas plataformas SCIELO, PubMed, Lilacs, Biblioteca Virtual de Saúde, com os descritores já mencionados inicialmente neste relato de caso. Foram utilizados artigos de língua inglesa, hispânica e portuguesa, datados de 2000 até 2018, com o objetivo de revisar e atualizar informações sobre a SCE, seu diagnóstico, evoluções em tratamento e casos semelhantes ao mencionando neste relato, entretanto não foram achados casos parecidos em nossas buscas.

Paciente feminina, 15 anos, natural e procedente de Franca, com relato de dor lombar, com início há três semanas, de moderada intensidade e com irradiação para ambos os membros inferiores, porém com predomínio à direita. Nos últimos 15 dias, apresentou intensificação gradativa da dor, sem alívio mesmo com o uso de analgésicos. Por fim, paciente evoluiu com disfunção urinária e fecal quando foi levada ao Pronto Socorro Municipal e encaminhada ao hospital de referência da cidade de Franca (interior do estado de São Paulo). Paciente previamente hígida, praticava atividade física supervisionada em academia da cidade.

A paciente foi admitida pelo serviço de neurocirurgia em regular estado geral, consciente, orientada no tempo e no espaço, corada, hidratada, pressão arterial $110 \times 80 \mathrm{mmHg}$, temperatura axilar de $36.6^{\circ} \mathrm{C}$. Aparelho cardiovascular: presença de bulhas rítmicas, normofonéticas em dois tempos e sem sopros, frequência cardíaca de 88 batimentos por minuto.

Aparelho respiratório: tórax simétrico, murmúrio vesicular presente e preservado globalmente sem ruídos adventícios, normopneico com a frequência respiratória de 19 incursões por minuto. Sinais neurológicos: dificuldade na marcha livre, deambulava com apoio, hipoestesia táctil e dolorosa na região perianal, hiporreflexia osteotendinosa patelar bilateral $(+)$ e diminuição do reflexo Aquileu bilateral $(+)$, força muscular dos membros inferiores sendo grau três no membro inferior direito (MID) e grau quatro no Membro Inferior Esquerdo (MIE).

Exame de ressonância magnética da coluna lombossacra evidenciou volumosa hérnia discal L5-S1 mediana (Figura 1). A paciente em questão foi, então, submetida a abordagem cirúrgica através de laminectomia no nível L5-S1 e remoção microcirúrgica do conteúdo discal herniado com descompressão radicular bilateral e do saco dural. Em seguida foi fixada com parafusos pediculares em L5 e S1 conectadas por barras longitudinais e artrodese póstero-lateral com enxerto autólogo procedente da lâmina e processo espinhoso.

Figura 1 - Ressonância Magnética evidenciando volumosa hernia discal entre L5-S1 mediana.

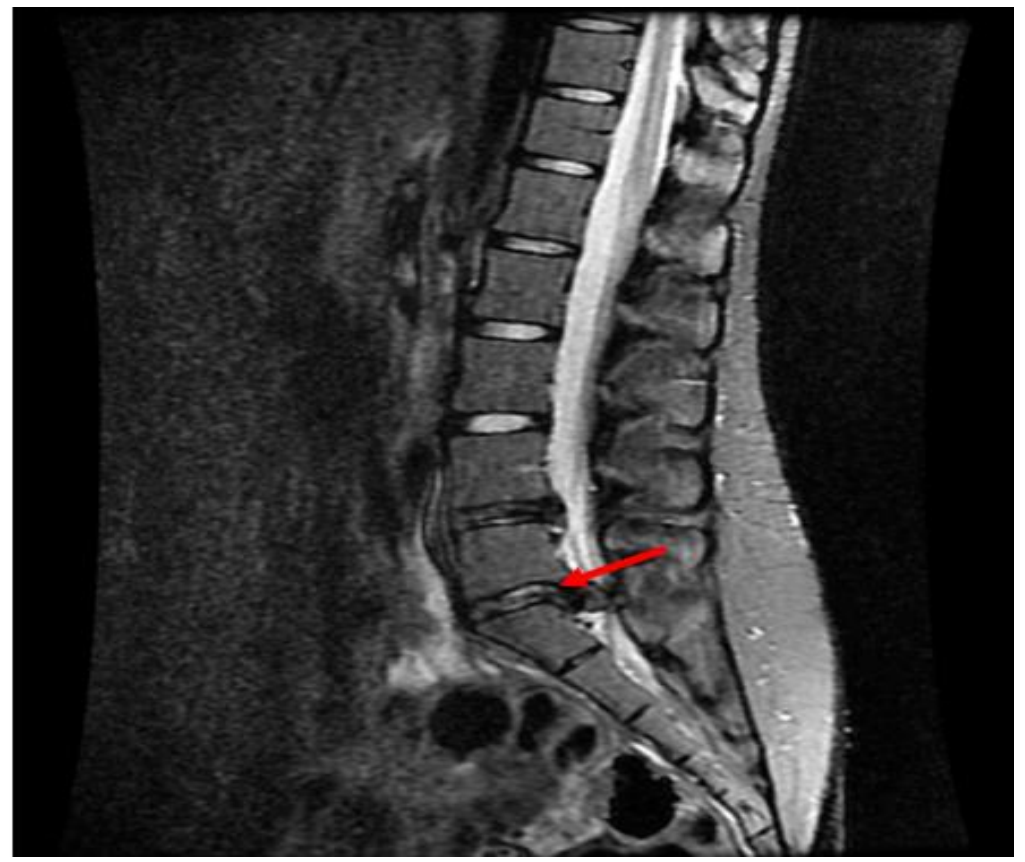

Fonte: Lima MO, et al., 2021. 
No pós-operatório imediato, ainda em nível hospitalar, iniciou a reabilitação com o serviço de fisioterapia neurológica da enfermaria. Na alta hospitalar, apresentava melhora relativa do quadro álgico e sensitivo, força muscular de ambos os membros inferiores, grau quatro, porém, persistia com quadro de incontinência urinária. Encaminhada para o tratamento fisioterápico e uroginecológico em nível ambulatorial.

\section{DISCUSSÃO}

A Síndrome da Cauda Equina cursa com dor lombar de forte intensidade, ciática bilateral, anestesia em sela (região interna da coxa, períneo e perianal), fraqueza motora nos níveis abaixo da compressão e disfunção esfincteriana, causando retenção ou incontinência fecal e urinária e disfunção sexual (PEREIRA CU, et al., 2013). Tal achado corrobora com o caso clínico em questão, em que a paciente, inicialmente, apresentava-se com quadro de dor lombar de intensidade moderada e irradiada para os membros inferiores de predomínio à direita e, posteriormente, intensificou-se gradativamente com o desfecho de disfunção urinária e fecal.

Os distúrbios intestinais e sexuais também podem fazer parte da apresentação clínica, porém é mais comum eles se tornarem presentes com a evolução do quadro, evidenciando uma consequência neurológica irreversível das raízes da cauda equina (TODD NV e DICKSON RA, 2016). Observa-se duas hipóteses que justificam a patogênese dessa doença, as quais estão em volta da compressão mecânica ou da isquemia da cauda equina e, observa-se que a compressão anatômica de raízes nervosas, geralmente por uma hérnia de disco vertebral em região lombar é uma causa comum na população geral (YANG SD, et al., 2017).

O nível mais comum de hérnia discal lombar que ocasiona SCE é L4-L5, seguido de L5-S1 e L3-L4 (CHAU AM, et al., 2014). A paciente do caso clínico em questão apresentou a sintomatologia compatível com a SCE devido uma hernia de disco lombar localizada em nível de L5-S1, o que reforça os dados apontados na literatura.

O início da doença pode ser agudo, dentro de horas, ou de evolução gradual, ao longo de semanas e meses (HAUPTFLEISCH J, et al., 2013). Além disso, é importante determinar se a SCE é completa, quando há incontinência esfincteriana indolor, ou incompleta, com alguma função esfincteriana preservada (PEREIRA CU, et al., 2013).

Não é necessária a presença de todos os achados clínicos simultaneamente, uma vez que os sinais clínicos não são sempre evidentes (BALASUBRAMANIAN K, et al., 2010). Diante disso, é necessário a realização de exames complementares como tomografia computadorizada (TC) e, o padrão-ouro, a ressonância magnética (RM) (HAUPTFLEISCH J, et al., 2013).

Diante da sintomatologia apresentada pela paciente citada, a equipe de atendimento seguiu os protocolos de diagnostico, visto que a confirmação diagnóstica é clínico-radiológico. Por essa razão, foi realizado o exame de ressonância magnética da coluna Lombo Sacra, o qual evidenciou volumosa hérnia discal L5-S1 mediana. A RM é necessária para a triagem primária do paciente com suspeita de SCE por causa da baixa sensibilidade da avaliação clínica e é tida como padrão ouro devido sua alta sensibilidade e especificidade dos tecidos moles, além de ser melhor quando comparada com a TC (HAUPTFLEISCH J, et al., 2013).

Embora a TC esteja disponível em quase todos os hospitais de cidades de médio e grande porte e, comumente, a ressonância magnética não, ela não conseguirá diagnosticar grandes prolapsos centrais do disco, nem tão pouco um hematoma epidural espinhal ou um abcesso espinhal, os quais são possiveis diagnósticos diferenciais para esses pacientes (GERMON T, et al., 2015).

A síndrome da cauda equina é considerada uma emergência neurocirúrgica, devido ao fato de que sua descompressão precoce proporciona redução de déficits neurológicos permanentes e diminui as complicações pós-operatórias (PEREIRA CU, et al., 2013).

O tratamento cirúrgico tem como objetivo descomprimir estruturas neurais, promover o alívio da dor, a recuperação e preservação neurológica e o restabelecimento funcional, além de ser a única maneira segura de impedir a progressão da lesão e de déficits neurológicos permanentes (BELL DA, et al., 2007). 
A descompressão deve ser realizada dentro das primeiras 48 horas, pois ela é o único fator que está diretamente relacionado com os melhores resultados. (CROCKER M, et al., 2008). Entretanto, mesmo que passadas as primeiras 48 horas a recomendação é de realizar a descompressão cirurgia, pois observa-se que muitos pacientes os quais foram submetidos a esse procedimento após o período considerado ideal, obtiveram sucesso e até recuperação completa (CROCKER M, et al., 2008).

Em contrapartida, alguns autores relataram que a descompressão cirúrgica precoce está indicada para paciente com lesão incompleta da SCE, para ajudar a progressão da doença para síndrome completa da SCE (PEREIRA CU, et al., 2013)

Então, em situações em que a SCE é diagnosticada, a cirurgia deverá ocorrer de forma precoce para minimizar os déficits neurológicos e beneficiar o prognóstico do paciente visto que qualquer atraso no tratamento só agrava a recuperação neurológica com a possibilidade de continuar com danos as raízes nervosas laterais que podem ter sobrevivido ao prolapso (FUSO FA, et al., 2013). Assim, como recomendado pela literatura, a paciente foi submetida à abordagem cirúrgica através de laminectomia no nível L5-S1 bilateral e artrodese, com estabilização do quadro clínico.

Dessa forma, o tratamento instituído no caso em questão teve como objetivo o alívio da dor, a recuperação e preservação neurológica e o restabelecimento funcional. No pós-operatório imediato, ainda em nível hospitalar, teve início a reabilitação com o serviço de fisioterapia neurológica da enfermaria, o qual também contribui para o melhor prognóstico da enfermidade. $\mathrm{Na}$ alta hospitalar, a paciente citada apresentava melhora relativa do quadro álgico e sensitivo, força muscular de ambos os membros inferiores grau quatro, porém, persistia com quadro de incontinência urinária com necessidade de manter o tratamento fisioterápico e uroginecológico em nível ambulatorial.

Todos os pacientes submetidos a cirurgia para estabilização do quadro devem ser orientados sobre o objetivo do tratamento e que há a possibilidade de desfechos melhores, mas deve ser esclarecido que existe um pequeno risco de piora do quadro, incluindo paralisia das pernas, perda completa do controle vesical e intestinal, além de impotência sexual (LICH NG, et al., 2004).

O prognóstico dessa enfermidade é determinado pelos sintomas de sua admissão e varia de acordo com a gravidade do distúrbio esfincteriano no pré-operatório, a extensão da diminuição da sensibilidade da bexiga e o grau de severidade da diminuição sensitiva na área perianal (GARDNER A, et al., 2011). É considerado um péssimo prognostico para a recuperação desses pacientes quando o diagnóstico é feito tardiamente, seja por desconhecimento do quadro ou confusão diagnóstica (QURESHI A e SELL P, 2007). Como consequência de um diagnóstico tardio, temos um tratamento tardio e, dessa forma, é possível concluir que a descompressão precoce, dentro de 48 horas, está associada a um melhor desfecho (BECULIC H, et al., 2016).

Após a revisão da literatura realizada nesse trabalho, foi possível concluir que a SCE é uma patologia rara, principalmente em populações jovens, correspondendo a uma emergência neurocirúrgica, cujo tratamento de eleição é a cirurgia descompressiva, visando um melhor desfecho clínico na tentativa de minimizar ou erradicar possíveis sequelas. Com isso, esse trabalho contribuiu significativamente para as pesquisas científicas, haja visto que se trata de um caso raro e pouco descrito na literatura, tendo como objetivo ajudar os outros profissionais da área da saúde a estudar e conduzir casos da doença relatada em paciente jovem. Além disso, tem-se um benefício adicional também aos pacientes portadores da doença que necessitam de tal abordagem terapêutica.

\section{REFERÊNCIAS}

1. PEREIRA CU, et al. Síndrome da cauda eqüina devido à hérnia discal lombar. Apresentação de caso. Revista Brasileira de Neurologia e Psiquiatria, 2013; 17(3): 41-48

2. VIALLE LR, et al. Lumbar disc herniation. Revista brasileira de ortopedia, 2010; 45(1): 17-22.

3. YANG SD, et al. Analysis of clinical and neurological outcomes in patients with cauda equina syndrome caused by acute lumbar disc herniation: a retrospective-prospective study. Oncotarget, 2017; 8(48): 84204. 
4. FUSO FA et al. Estudo epidemiológico da síndrome da cauda equina. Acta ortop. bras, 2013; 21(3): 159-162.

5. GARDNER A, et al. Cauda equina syndrome: a review of the current clinical and medico-legal position. European Spine Journal, 2011; 20(5): 690.

6. CHAU AMT, et al. Timing of surgical intervention in cauda equina syndrome: a systematic critical review. World neurosurgery, 2014; 81(4):640-650.

7. AHN UM, et al. Cauda equina syndrome secondary to lumbar disc herniation: a meta-analysis of surgical outcomes. Spine, 2000; 25(12): 1515-1522.

8. GITELMAN A, et al. Cauda equine syndrome: a comprehensive review. Am J Orthop 2008; 37(1): 56-62

9. CROCKER M, et al. The value of interhospital transfer and emergency MRI for suspected cauda equina syndrome: a 2-year retrospective study. The Annals of The Royal College of Surgeons of England, 2008; 90(6): 513-516.

10. TODD NV, DICKSON RA. Standards of care in cauda equina syndrome. British journal of neurosurgery, 2016; 30(5): 518-522.

11. HAUPTFLEISCH J, et al. Out-of-hours MRI provision in the UK and models of service delivery. Clinical radiology, 2013; 68(5): e245-e248.

12. BALASUBRAMANIAN K, et al. Reliability of clinical assessment in diagnosing cauda equina syndrome. British journal of neurosurgery, 2010; 24(4): 383-386.

13. GERMON T, et al. British Association of Spine Surgeons standards of care for cauda equina syndrome. The Spine Journal, 2015; 15(3): S2-S4.

14. BELL DA, et al. Cauda equina syndrome-What is the correlation between clinical assessment and MRI scanning. British journal of neurosurgery, 2007; 21(2): 201-203.

15. LICH NG, et al. Cauda equina syndrome: an audit. Can we do better?. Journal of Orthopaedic Medicine, 2004;26(3): 98-101.

16. QURESHI A, SELL P. Cauda equina syndrome treated by surgical decompression: the influence of timing on surgical outcome. European Spine Journal, 2007; 16(12): 2143-2151.

17. BEČULIĆ H, et al. Impact of timing on surgical outcome in patients with cauda equina syndrome caused by lumbar disc herniation. Med Glas, 2016; 13(1):136-141. 